“C 2016 IEEE. Personal use of this material is permitted. Permission from IEEE must be obtained for all other uses, in any current or future media, including reprinting/republishing this material for advertising or promotional purposes, creating new collective works, for resale or redistribution to servers or lists, or reuse of any copyrighted component of this work in other works." 


\title{
Experimentally Validated, Planar, Wideband, Electrically Small, Monopole Filtennas Based on Capacitively Loaded Loop Resonators
}

\author{
Ming-Chun Tang, Senior Member, IEEE, Ying Chen, and Richard W. Ziolkowski, Fellow, IEEE
}

\begin{abstract}
Two planar, efficient, wideband, electrically small, monopole filtennas are presented. The first one evolved directly from a common planar capacitively loaded loop (CLL)-based filter. This filtenna possesses a flat realized gain response within the operational band and good band-edge selectivity. The second filtenna consists of a driven element augmented with a CLL structure and with slots etched into its ground plane. It expands the fractional impedance bandwidth of the first case from $6.28 \%$ up to $\mathbf{7 . 9 \%}$. It too has a gain response that remains flat over its operational bandwidth and even higher band-edge selectivity. Both filtennas are electrically small: $k a<1$. The experimental results, which are in good agreement with their simulated values, demonstrate that both filtennas exhibit excellent impedance matching, high radiation efficiency, flat gain response, and steep skirts at both band-edges, as well as producing monopole radiation patterns that are uniform and nearly omni-directional in their H-planes.
\end{abstract}

Index Terms-Bandwidth, electrically small antenna, efficient, planar antenna, near-field resonant parasitic element, filtenna

\section{INTRODUCTION}

Filsin iltennas are co-designed antennas that combine a radiating element and a filter into a single module. Because of its self-contained filtering function, a filtenna has three advantages over common antennas when a signal is received. First, it reduces the interconnection losses that occur when a standard receiving antenna is connected to a filter in the processing layer. Second, it suppresses unwanted signals out of the operational band. Third, from a practical point of view, it facilitates a more

Manuscript received on Mar. 30, 2016; revised on May 31, 2016; accepted on June 01, 2016.

This work was supported in part by the National Natural Science Foundation of China contract number 61471072, in part by the Fundamental Research Funds for the Central Universities contract number 106112015CDJZR165510, in part by China Postdoctoral Science Foundation contract number 2016M590860, and in part by Opening subject of State Key Laboratory of Millimeter Waves contract number K201732.

M. -C. Tang is with the College of Communication Engineering, Chongqing University, Chongqing, 400044, China and with the State Key Laboratory of Millimeter Waves, Nanjing, 210096, China (E-mail: tangmingchunuestc@126.com);

Y. Chen is with the College of Communication Engineering, Chongqing University, Chongqing, 400044, China;

R. W. Ziolkowski is with the Department of Electrical and Computer Engineering, University of Arizona, Tucson, AZ 85721 USA and with the Global Big Data Technologies Centre, University of Technology Sydney, Ultimo NSW 2007, Australia (E-mail: ziolkowski@ece.arizona.edu). compact and lower costs RF front-end system. As a consequence, a variety of filtennas have been developed. Examples include filtering horn antennas [1]-[3], substrate integrated waveguide (SIW) filtennas [4], [5], planar filtering ultra-wideband (UWB) antennas [6], [7], filtering microstrip patch antennas [8]-[11], filtering slot antennas [12], and filtering quasi-Yagi antennas [13].

Efforts to miniaturize and compactify filtennas have employed composite right/left handed transmission lines (CRLH-TLs) [14], [15] and multiple electrically small resonators in series [16], [17]. Unfortunately, the relatively large radiation patch / long meander-line strip above the ground required in those CRLH-TL-based filtenna designs prohibits any further miniaturization [14], [15]. Similarly, multi-resonator filtenna makes use of multi-pole filter design principles, exploiting the presence of more transmission poles to achieve broader operational bandwidths. This inescapably increases their electrical sizes [16], [17], which are deemed to be not small, i.e., $k a>1$ (where $a$ is the radius of the smallest sphere that completely encloses the antenna at the lowest operational frequency, $f_{L}$, and $k=2 \pi / \lambda_{0}=2 \pi f_{L} / \mathrm{c}$ is the corresponding free space wave number) [14]-[17].

In this paper, we report two electrically small, efficient, planar, monopole filtennas based on capacitively loaded loop (CLL) resonators. First, the performance characteristics of filters that are based on a pair of electrically coupled CLL resonators are analyzed in Section II. With the aid of the corresponding equivalent lumped-element circuit models, their responses in uncoupled, low coupling and strong coupling configurations are demonstrated and compared analytically and numerically. Knowing these features, we designed, fabricated, and measured the first filtenna. As discussed in Section III, the experimental results demonstrate that this electrically small system had a $6.27 \%$ fractional impedance bandwidth, high out-of-band rejection, and stable omnidirectional radiation patterns. The analysis of the current distributions on its metallic surfaces confirmed our filtenna design methodology. A second filtenna was then designed, fabricated, and measured. Additional CLL structures, as near-field resonant parasitic (NFRP) elements are now organically integrated into the system to achieve a wider operational bandwidth. The resulting filtenna has a $7.9 \%$ fractional impedance bandwidth, together with a flat 
gain response, stable omni-directional radiation patterns, and high out-of-band rejection characteristics.

We note that all of the metallic elements modeled in the antenna designs were chosen to be copper with its known material parameters: $\varepsilon_{r}=1.0, \mu_{r}=0.999991$ and bulk conductivity $\sigma=5.8 \times 10^{7} \mathrm{Siemens} / \mathrm{m}$. All of the numerical simulations and their optimizations were performed using the frequency domain, finite element-based ANSYS/ANSOFT High Frequency Structure Simulator (HFSS), version 13.0 [18].

\section{Design OF SQuARE CLL-BASEd BANDPASS FILTERS}

A microstrip rectangular CLL-based bandpass filter with a $0^{\circ}$ feed structure [19] is illustrated in Fig. 1(a). The dashed lines $\mathrm{O}-\mathrm{O}^{\prime}$ and $\mathrm{T}-\mathrm{T}^{\prime}$ represent its symmetrical planes along the $x$-axis and $y$-axis, respectively. The Rogers 4350B substrate, with permittivity $\varepsilon_{r}=3.48$, permeability $\mu_{r}=1$, and dielectric loss tangent $\tan \sigma=0.0037$, was selected to construct the filter. Its total size was $29 \mathrm{~mm} \times 27 \mathrm{~mm} \times 1 \mathrm{~mm}$. In the design, two square CLLs were etched on the substrate and were oriented with their gaps face-to-face. This configuration produces an electrical coupling between the two elements [19]-[21], which has been utilized previously, for example, to significantly improve the transmission of microwave fields through a metallic subwavelength aperture [22]. The two feeding ports of the filter shown in Fig. 1(a), which are connected to $50 \Omega$ microstrip lines (with the width $2.2 \mathrm{~mm}$ ), are positioned at the opposite sides of the line $\mathrm{O}-\mathrm{O}^{\prime}$ in order to create two extra transmission zeros in the stop-band while the passband response remains unchanged. As reported in [19], this $0^{\circ}$ feed arrangement is superior to the one having them on the same side.

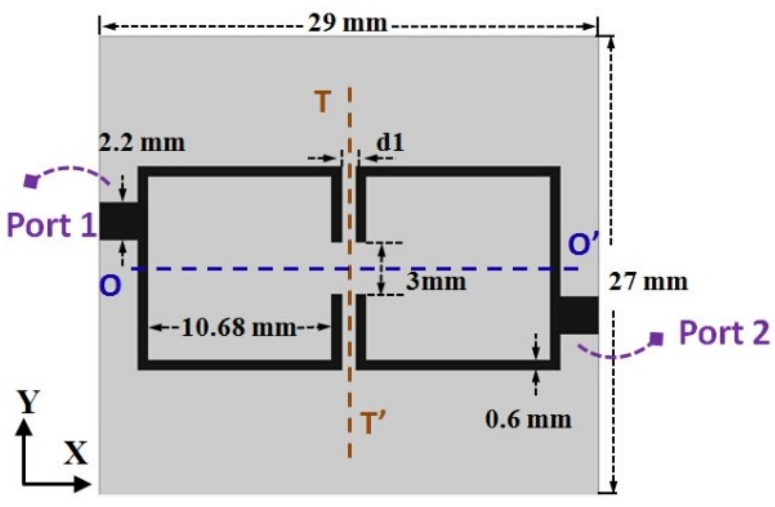

(a)

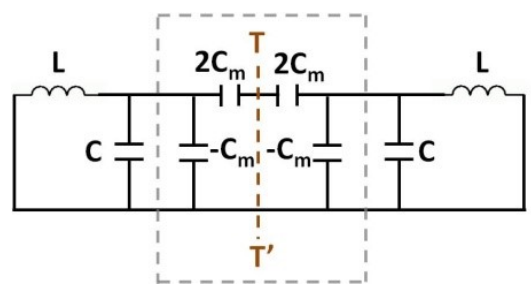

(b)

Fig. 1 The filter with electrically coupled resonators. (a) The design layout, and (b) its equivalent circuit network [20].
The corresponding equivalent lumped-element circuit is given in Fig. 1(b). Its $\mathrm{L}$ and $\mathrm{C}$ values are the ideal self-inductance and self-capacitance of the uncoupled resonators, respectively. The term $\mathrm{C}_{\mathrm{m}}$ is the mutual capacitance of the two resonators. When the symmetry plane $T-T^{\prime}$ is replaced by an electric wall (a short circuit), the resultant circuit has a lower resonance frequency $f_{e}=1 /\left\{2 \pi\left[\mathrm{L}\left(\mathrm{C}+\mathrm{C}_{\mathrm{m}}\right)\right]^{1 / 2}\right\}$. Similarly, when it is replaced by a magnetic wall (an open circuit), the resultant circuit has a higher resonance frequency $f_{m}$ $=1 /\left\{2 \pi\left[\mathrm{L}\left(\mathrm{C}-\mathrm{C}_{\mathrm{m}}\right)\right]^{1 / 2}\right\}[19]$.

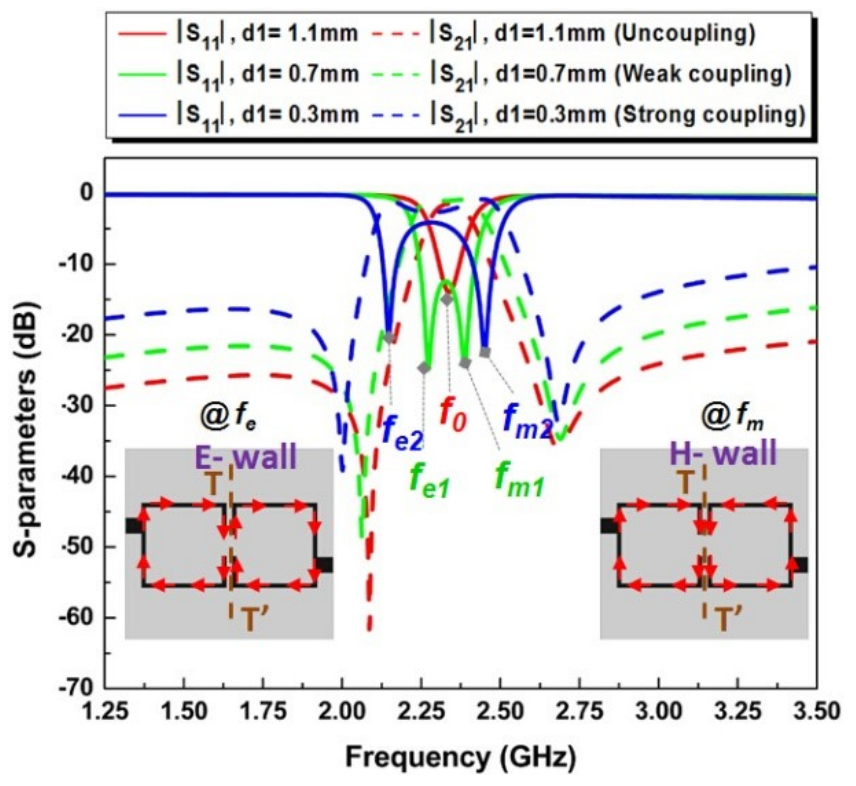

Fig. 2 Simulated S-parameters of the electrically coupled filter when the distance of separation $\mathrm{d} 1$ is varied from $1.1 \mathrm{~mm}$ to $0.3 \mathrm{~mm}$, together with the surface current distribution behaviors on the resonators at $f_{e}$ and $f_{m}$.

The simulated S-parameters of the filter, when the distance between the two resonators (d1) is varied from 1.1 to $0.3 \mathrm{~mm}$, are presented in Fig. 2. Our simulations show that when the distance $\mathrm{d} 1$ becomes large enough (e.g., greater than $1.1 \mathrm{~mm}$ ), the resonance frequency $f_{0}$ keeps unchanged, and the resonance strength $\left(\left|\mathrm{S}_{11}\right|\right.$ dip$)$ at $f_{0}$ changes little. Since the mutual coupling between the resonators is negligible, it does not impact the resonance frequency $f_{0}$ and the mutual capacitance is approximately zero, i.e., $\mathrm{C}_{\mathrm{m}} \approx 0$. Thus, in this approximate uncoupled case, $f_{e} \approx f_{m} \approx f_{0}=1 /\left[2 \pi(\mathrm{LC})^{1 / 2}\right]$ is obtained. In contrast, when the distance d1 becomes close enough (e.g., lower than $0.7 \mathrm{~mm}$ ), the resonance frequency $f_{0}$ is completely split into two nearby frequencies, i.e., $f_{e}$ and $f_{m}$, to create a wider passband. It was observed from our simulations that at the lower resonance frequency, $f_{e}$, the surface currents on the two loops are in-phase. This result agrees with the choice of the electric wall for the plane labeled $\mathrm{T}-\mathrm{T}^{\prime}$. On the other hand, the surface currents on the two CLLs are out-of-phase at the higher resonance frequency, $f_{m}$. This result agrees with the choice of the magnetic wall for the plane labeled $\mathrm{T}-\mathrm{T}^{\prime}$. Moreover, by further decreasing the distance $\mathrm{d} 1$, the mutual coupling becomes even stronger, leading to an even larger $\mathrm{C}_{\mathrm{m}}$ value. Thus, both $f_{e}$ and $f_{m}$ appear further away from $f_{0}$, as is shown in Fig. 2. 
Therefore, with a narrow gap size $\mathrm{d} 1$ for the filter structure in Fig. 1(a), one finds a much stronger coupling between the CLL resonators; and this causes the corresponding two poles to repel one another, resulting in a much wider bandwidth.

This can be verified by calculating the electric coupling coefficient $\left(k_{e}\right)$ between two resonators. It is readily obtained using the expression [20]:

$$
k_{e}= \pm \frac{1}{2}\left(\frac{f_{2}}{f_{1}}+\frac{f_{1}}{f_{2}}\right) \sqrt{\left(\frac{f_{m}^{2}-f_{e}^{2}}{f_{m}^{2}+f_{e}^{2}}\right)^{2}-\left(\frac{f_{2}^{2}-f_{1}^{2}}{f_{2}^{2}+f_{1}^{2}}\right)^{2}}
$$

where $f_{1}$ and $f_{2}$ indicate the resonance frequencies of each independent CLL resonator. Because the capacitance $C_{m}$ in Fig. 1, is positive, the plus sign is selected. Furthermore, because the two CLL resonators are identical, one knows that $f_{1}$ $=f_{2}$. Thus, Eq. (1) reduces to the following:

$$
k_{e}=\frac{f_{m}^{2}-f_{e}^{2}}{f_{m}^{2}+f_{e}^{2}}
$$

Consequently, as expected for the uncoupled, weak coupling, and strong coupling cases given in Fig. $2, k_{e}$ is, respectively, 0 , 0.14 , and 0.49 .

\section{Design of Electrically Small FiltenNa}

Based on the electrically coupled filter configuration in Fig. 1, a filtenna having a second-order filter was co-designed and optimized. The Rogers 4350B substrate, which had the same size and thickness used for the filter in Fig. 1, was selected for this filtenna design. The final result is shown in Figs. 3(a) and 3 (b); the corresponding optimized design parameters are listed in Table I. The fabricated prototype is shown in Fig. 3(c).

As shown in Fig. 3(a), one CLL element (half of the filter shown in Fig. 1) acts as the driven element. As shown in Fig. 3(b), it lies on the upper surface of the Rogers copper-cladded sheet. The lower surface simply has a ground strip etched out of it. The CLL element is directly fed from the source via a $50 \Omega$ microstrip line. A fan-shaped radiator has been substituted for the other CLL element. It acts as a resonant parasitic element in the near field of the monopole (CLL-based) antenna [23]. This special fan-shaped radiator choice is made to establish an even smoother impedance transition over the desired wider bandwidth. Moreover, because the second CLL element has been replaced by the fan-shaped radiator, their individual resonant frequencies (i.e., $f_{1}$ and $f_{2}$ ) must be determined separately and tuned individually to be close to each other, but not necessarily equal. While this choice invalidates the simple expression (2) to calculate the coupling coefficient, it nonetheless provides an additional degree of freedom in the design process. Simply starting with the resonance frequencies near to each other facilitates a straightforward numerical approach to finalize the actual antenna design.

Note that the fan-shaped part of the NFRP element is placed on the opposite side of the reference line $\mathrm{O}-\mathrm{O}^{\prime}$ with respect to the position of the feed port. This arrangement corresponds to the port assignments of the filter shown in Fig. 1 and creates dual transmission zeros on the two edges of the passband, which enhance the out-of-band rejection level. The fan feature has no portion of the ground plane under it.

The straight-line section along the $y$-direction (with length W7) of the NFRP element is close to the gap of the CLL and is constructed to be capacitively coupled to it, the driven element. Hence, it yields the equivalent of the coupling capacitance $\mathrm{C}_{\mathrm{m}}$. However, due to the absence of majority of the ground strip underneath this parasitic element, the coupling level is not strong enough to excite it. Therefore, a second straight-line strip (with length L5) along the $x$-direction under the driven element was added not only to improve the capacitive coupling level, but also to increase the total length $(\sim 45.9 \mathrm{~mm})$ to be close to the length of the driven CLL element $(\sim 43.4 \mathrm{~mm})$. We note that even though it is not shown in Fig. 3, a short coaxial cable was actually included in the HFSS model to enhance the agreement between the simulated and experimental results.

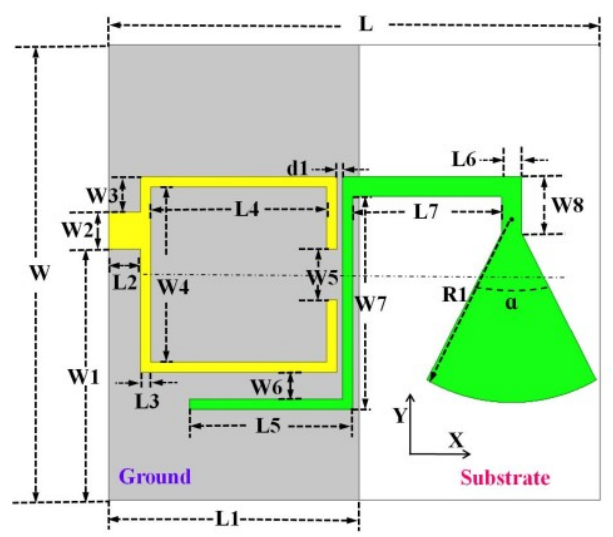

(a)

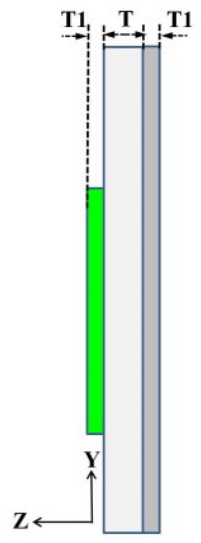

(b)

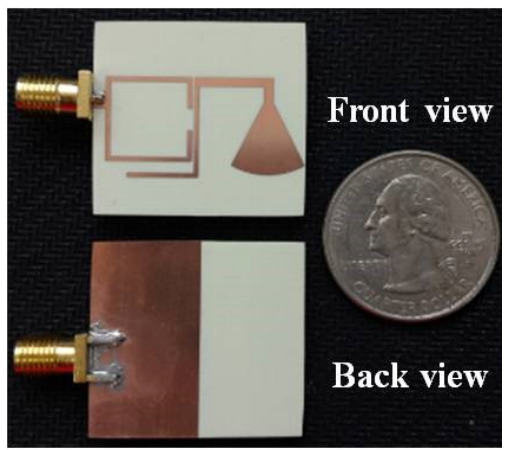

(c)

Fig. 3 The first electrically small filtenna. (a) Top view and (b) side view of the HFSS model, and (c) the front and back views of the fabricated prototype.

TABle I. The Optimized Design PARAmeters of the First FiltennA (All Dimensions ARE IN MiLlimeters)

\begin{tabular}{|c|c|c|c|c|}
\hline$L=29$ & $L 1=14.77$ & $L 2=1.87$ & $L 3=0.6$ & $L 4=10.4$ \\
\hline$L 5=9.6$ & $L 6=1.2$ & $L 7=8.8$ & $W=27$ & $W 1=14.9$ \\
\hline$W 2=2.2$ & $W 3=2.1$ & $W 4=10.4$ & $W 5=3$ & $W 6=1.6$ \\
\hline$W 7=12.6$ & $W 8=3.4$ & $R 1=10$ & $\alpha=60^{0}$ & $T=1$ \\
\hline$T 1=0.017$ & $d 1=0.31$ & \multicolumn{3}{|c|}{ Null } \\
\hline
\end{tabular}


The advantages of this arrangement were first confirmed by our simulation studies. The filtenna was then fabricated and measured. The reflection coefficient of the prototype system was obtained using an Agilent E8361A PNA Vector Network Analyzer (VNA). The measured $\left|\mathrm{S}_{11}\right|$ values, together with the simulated values, are presented in Fig. 4. The measured values confirmed that the filtenna had a $-10 \mathrm{~dB}$ impedance bandwidth (i.e., the set of frequencies for which $\left|\mathrm{S}_{11}\right|<-10 \mathrm{~dB}$ ) from 2.24 $\mathrm{GHz}$ to $2.385 \mathrm{GHz}$ (6.27\% fractional impedance bandwidth) in good agreement with the simulated values 2.252 to $2.398 \mathrm{GHz}$ (6.28\% fractional impedance bandwidth). The electrical size of the measured prototype was calculated to be $k a \sim 0.93$, while its simulated value was $\sim 0.935$.

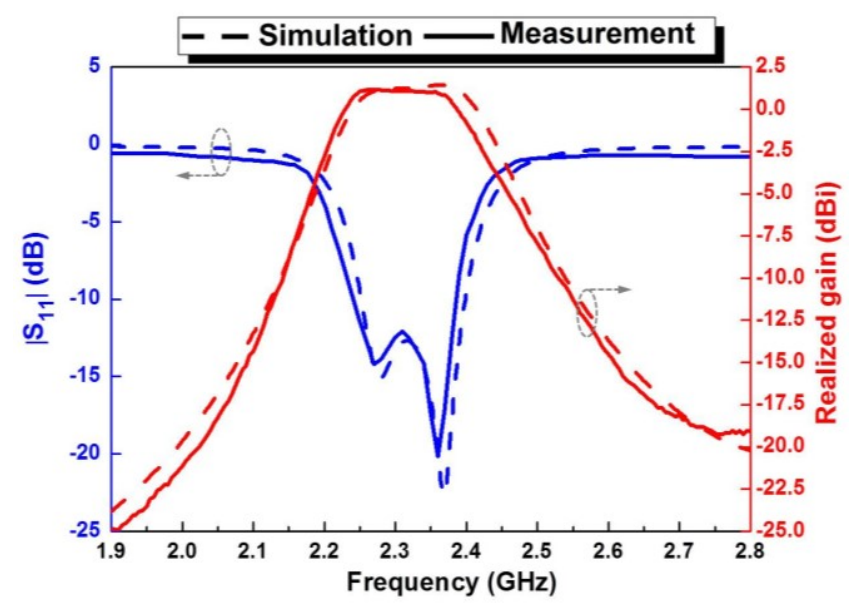

Fig. 4 The measured and simulated $\left|S_{11}\right|$ and realized gain responses of the first filtenna as functions of the source frequency.

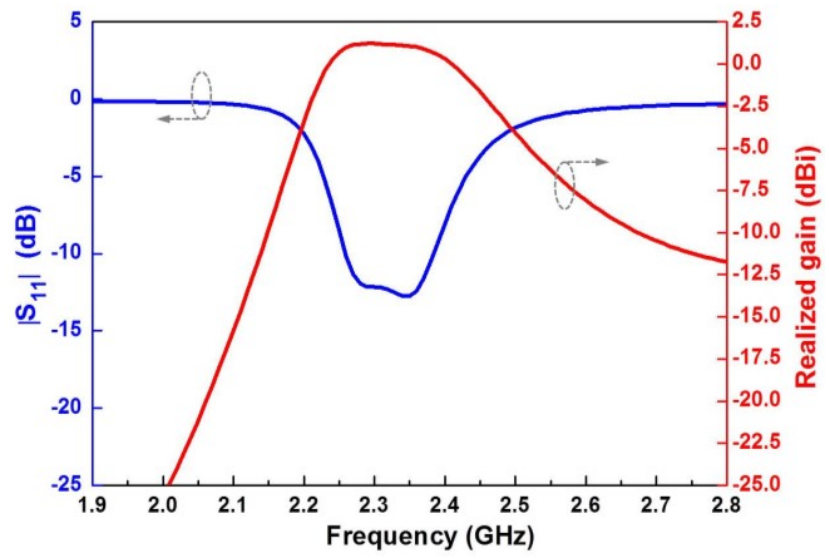

Fig. 5 The simulated $\left|S_{11}\right|$ and realized gain responses of the comparison filtenna design with the fan-shaped segment on the same side of the reference line $\mathrm{O}-\mathrm{O}^{\prime}$ with respect to the feed port as functions of the source frequency.

The far-field radiation performance characteristics of the filtenna were also studied numerically and experimentally. The experiments were carried out in an anechoic chamber at the National Key Laboratory of Science and Technology on Communications, University of Electronic Science and Technology of China (UESTC). This facility is mainly based on the Agilent N5230A PNA-L VNA and the SATIMO passive measurement system [24]. Both the experimental and simulated results indicated that the filtenna radiated a monopole-like pattern in the ZOX $(E-)$ plane and a nearly omni-directional pattern in the ZOY $(H-)$ plane. The experimental and simulated realized gain values along $+Z$-axis as a function of the source frequency are also provided in Fig. 4. They demonstrate that the prototype filtenna has a flat realized gain response within its passband, which has a measured (simulated) 1.15 (1.41) dBi peak value. The simulated radiation efficiency was higher than $80.93 \%$ throughout the operational band. Moreover, sharp slopes are observed at both the upper and lower edges of the passband. They indicate that the first realized filtenna has very good band-edge selectivity and stop-band suppression.

In order to emphasize the importance of placing the fan-shaped segment of the NFRP element on the opposite side of the reference line $\mathrm{O}-\mathrm{O}^{\prime}$ with respect to the feed port, the filtenna with it on the same side (i.e., W3 $=7.9 \mathrm{~mm}$ ) was simulated. These comparison results are given in Fig. 5. They demonstrate that the stop-band suppression performance on the upper edge is degraded significantly with this choice.

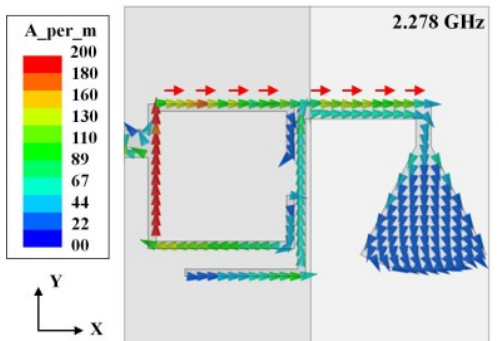

(a)

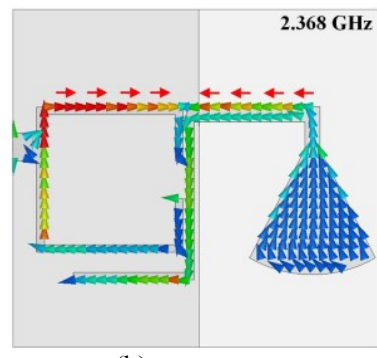

(b)
Fig. 6 The current distributions on the first filtenna (a) at the lower resonance frequency, $2.278 \mathrm{GHz}$, and (b) at the higher resonance frequency, $2.368 \mathrm{GHz}$.

Fig. 6 gives the HFSS predicted current distributions on the upper surfaces of the driven CLL and NFRP elements of the first filtenna at its two resonance frequencies. As expected from its design, these resonances correspond to the two transmission poles of the filter shown in Fig. 2. One observes that the current flow along the driven CLL element, as well as along the vertical ( $y$-axis) strip of the NFRP element, conforms to the behavior shown in Fig. 2. As is indicated by the red arrows, the currents on the two straight upper horizontal strips of the CLL and NFRP elements are oriented along the $x$-direction: along the CLL strip and along the top edge of the NFRP segment. Again, in correspondence with Fig. 2, these particular current segments are in-phase at the lower resonance frequency and out-of-phase at the higher one.

\section{FILTENNA WITH ENHANCED BANDWIDTH}

While the electrically small filtenna reported in Section III exhibits good radiation and filtering performances, it would be desirable for many applications to have more bandwidth. In order to achieve a wider bandwidth while maintaining its overall electrically small size $(k a<1)$, a variation of the first filtenna was considered. Its design is shown in Fig. 7. The corresponding optimized design parameters are listed in Table 
II. Again, the same size and thickness Rogers 4350B substrate was selected to construct this filtenna.

Recalling our previous filter discussion, when the two resonators get physically closer to each other, their stronger mutual coupling causes its resonance frequency $f_{e}\left(f_{m}\right)$ to become lower (higher). Consequently, because these resonances move away from each other, more energy is reflected and less power is transmitted in the frequency range from $f_{e}$ to $f_{m}$. This corresponds to the appearance of the dip in the $\left|\mathrm{S}_{21}\right|$ curve in Fig. 2 for the $\mathrm{d} 1=0.3 \mathrm{~mm}$ case.

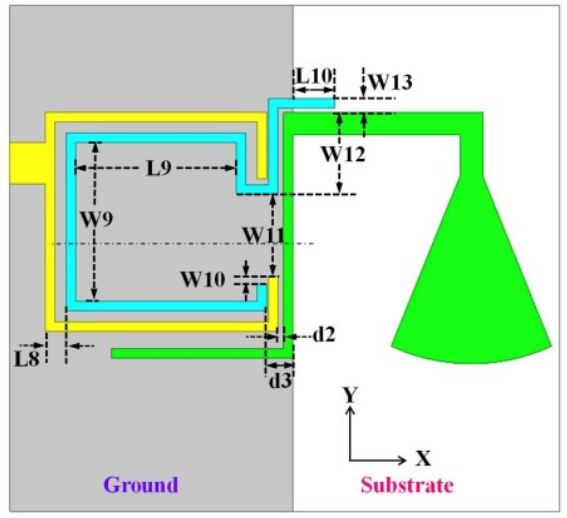

(a)

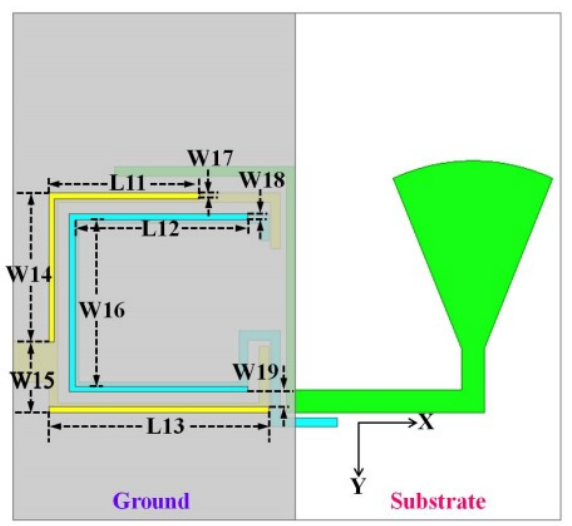

(b)

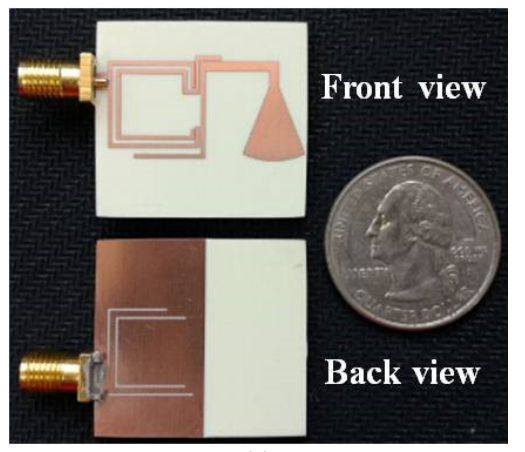

(c)

Fig. 7 The enhanced bandwidth filtenna with slots in its ground strip. (a) Top view, (b) back view, and (c) the front and back views of the fabricated prototype.

In order to improve the flatness of the transmission performance within the passband while maintaining its wideband operation and steep skirts, a third resonator was introduced whose resonance frequency lies near the middle of $f_{e}$ and $f_{m}$ and whose bandwidth does not overlap with either.

The third resonator is the additional CLL element (in blue) shown in Fig. 7(a). Its gap coincides with the gap of the driven CLL element, and it has an arm that has been included so that it also couples to the NFRP element. This co-located arrangement of the two CLLs, as analyzed in detail and applied in our previous UWB antenna and magnetic metamaterial studies [25, 26], provides a means to control the mutual coupling, further expanding the bandwidth. Moreover, unlike the reported design technology [17] of placing several resonators in series to achieve a broader operational bandwidth, this arrangement does not increase the total overall dimensions of the filtenna.

It was desired that the length of the additional CLL element be set nearly equal to the driven CLL's value to make their resonance frequencies close to one another. However, because it lies inside the driven element, the length of additional CLL element had to be increased. This was accomplished by adding a segment of microstrip line outside of the driven element. The resulting optimized total length was $43.02 \mathrm{~mm}$ in contrast to the $42.12 \mathrm{~mm}$ length of the driven CLL. Furthermore, in order to balance the mutual coupling levels amongst the two CLLs and the NFRP element, the path of this extra strip was fixed parallel to one vertical segment of the driven CLL and near to the horizontal segment (its strip that is orthogonal to the wide ground strip) of the NFRP element.

TABle II. ThE OPtIMIZED Design PARAMETERS OF THE FILTENNA With ENHANCED BANDWIDTh (All DimENSIONS ARE In MiLlimeters)

\begin{tabular}{|c|c|c|c|c|}
\hline$L=29$ & $L 1=14.95$ & $L 2=1.87$ & $L 3=0.5$ & $L 4=10.68$ \\
\hline$L 5=9.6$ & $L 6=1.2$ & $L 7=8.8$ & $L 8=1.1$ & $L 9=8.48$ \\
\hline$L 10=2.2$ & $L 11=8$ & $L 12=9.18$ & $L 13=11.68$ & $W=27$ \\
\hline$W 1=17.48$ & $W 2=2.2$ & $W 3=1.6$ & $W 4=10.68$ & $W 5=5.2$ \\
\hline$W 6=0.9$ & $W 7=11.88$ & $W 8=3.4$ & $W 9=8.48$ & $W 10=0.4$ \\
\hline$W 11=2.2$ & $W 12=4.34$ & $W 13=0.5$ & $W 14=7.9$ & $W 15=3.48$ \\
\hline$W 16=8.88$ & $W 17=0.3$ & $W 18=0.3$ & $W 19=0.8$ & $R 1=10$ \\
\hline$\alpha=50^{\circ}$ & $T=1$ & $T 1=0.017$ & $d 1=0.9$ & $d 2=0.3$ \\
\hline$d 3=1.4$ & \multicolumn{5}{|c|}{ Null } \\
\hline
\end{tabular}

The simulated $\left|\mathrm{S}_{11}\right|$ and realized gain values are presented in Fig. 8. They clearly indicate that by adding the second CLL, the desired third pole was indeed created in the range between $f_{e}$ and $f_{m}$. Moreover, the $-10 \mathrm{~dB}$ impedance bandwidth was increased to $8.4 \%$. At the same time, the realized gain values were not decreased across the operational frequency band and the out-of-band rejection performance was maintained. However, a ripple (see the insert) with $\mathrm{a} \sim 0.25 \mathrm{~dB}$ fluctuation appears on the realized gain curve in the neighborhood of the second pole at $2.34 \mathrm{GHz}$. This feature was traced through simulation studies to arise from a small amount of electromagnetic energy that becomes trapped within the substrate between both CLL elements and the ground strip and is not radiated away from the structure. It is associated with the inherent high-Q behavior associated with double CLL structures like the split-ring resonator (SRR) $[27,28]$. This trapping lowers the radiation 
efficiency in the neighborhood of $2.34 \mathrm{GHz}$ and, hence, the realized gain there.

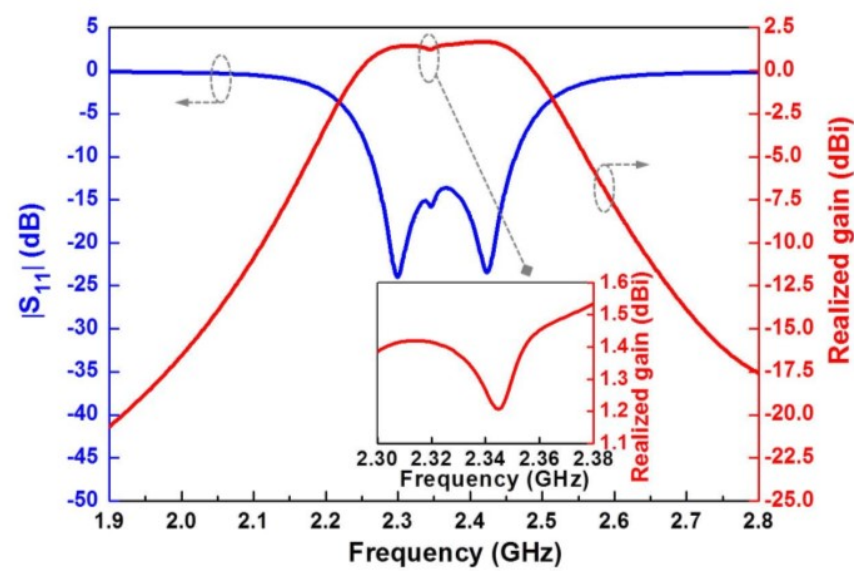

Fig. 8 Simulated $\left|S_{11}\right|$ and realized gain values as functions of the source frequency for the enhanced bandwidth filtenna without slots in its ground strip.

In order to eliminate the ripple, three slots were etched on the ground strip directly beneath the two CLL elements, as shown in Fig. 7(b). Notice that the gap (W15) between the two outer slots; it makes them disconnected. Because it maintains the same partition of the backside of the copper-cladded substrate into the ground strip underneath the feedline, this choice avoids any influence the slots might have on the impedance characteristics of the microstrip feedline, which lies on the other side of the substrate. The resulting current distribution on the ground strip at the additional resonance frequency is presented in Fig. 9. It demonstrates that a large amount of the surface currents is now tied to the slots, particularly the complete U-shaped one, and is subsequently radiated away from the structure as a result. The presence of the two outer slots facilitates the fine tuning of the input impedance at and near this resonance frequency.

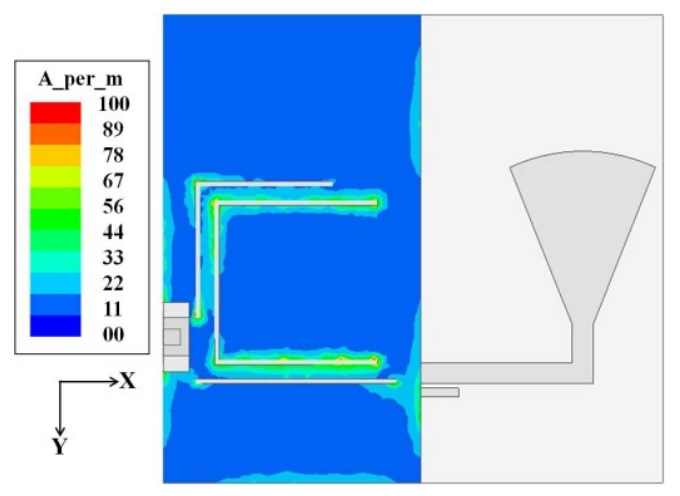

Fig. 9 The surface current distribution on the slotted ground strip of the enhanced bandwidth filtenna at $3.34 \mathrm{GHz}$, its middle resonance frequency.

The simulated and measured $\left|\mathrm{S}_{11}\right|$ and realized gain values of the second filtenna with the group strip slots are given in Fig. 10. The simulated (measured) realized gain values indicate that the ripple feature not only was significantly decreased to $\sim 0.03 \mathrm{~dB}$ $(\sim 0.11 \mathrm{~dB})$ in the neighborhood of the second pole at $2.34 \mathrm{GHz}$ $(2.30 \mathrm{GHz})$, but the peak realized gain also was increased from
1.659 to $1.75 \mathrm{dBi}$ in simulation. It was measured to be $1.376 \mathrm{dBi}$, indicating more losses were encountered than expected in the fabricated prototype. From the $\left|\mathrm{S}_{11}\right|$ values, the impedance bandwidth was simulated to be from 2.264 to $2.46 \mathrm{GHz}(8.3 \%$ fractional bandwidth), and was from 2.261 to $2.447 \mathrm{GHz}(7.9 \%$ fractional bandwidth) in the experiment, in very good agreement. Similarly, the simulated $k a \sim 0.94$ and measured $k a$ $\sim 0.938$ values agree and verify that the filtenna is electrically small. Furthermore, the simulated radiation efficiency across the entire operational bandwidth is higher than $82.87 \%$.

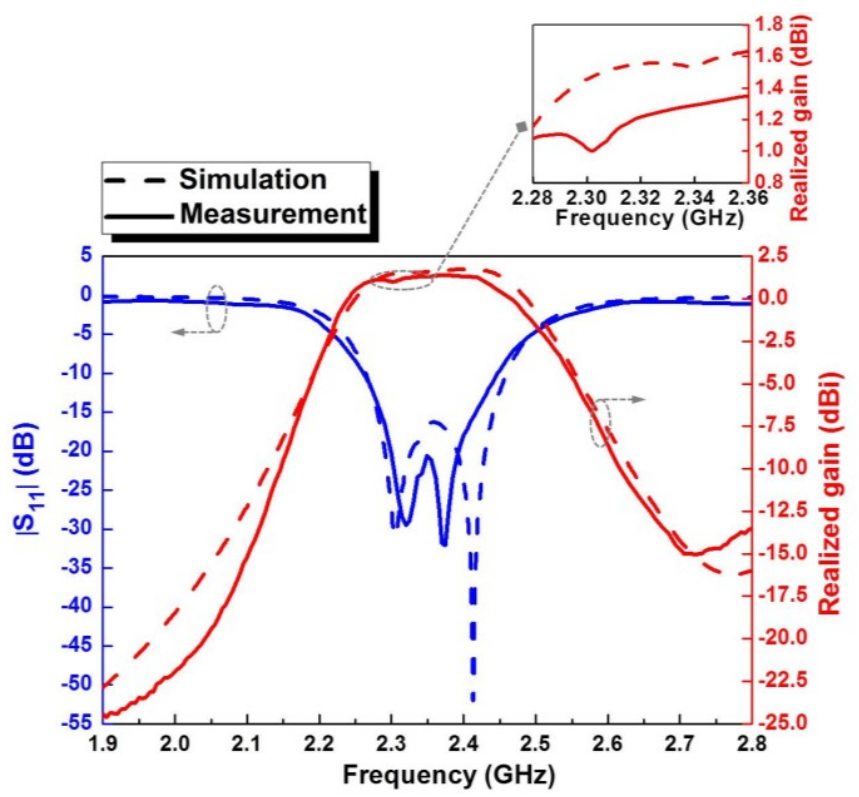

Fig. 10 The measured and simulated $\left|\mathrm{S}_{11}\right|$ and realized gain values as functions of the source frequency for the enhanced bandwidth filtenna with slots in its ground strip.

The simulated surface current distributions on the driven and parasitic elements are presented in Fig. 11. Clearly, these current distributions are, respectively, in-phase and out-of-phase at the lower and higher resonance frequencies, as is shown in Figs. 11(a) and (b). This surface current behavior matches that of the two-pole filtenna in Fig. 6 very well. Fig. 11(c) shows that the currents on the inner CLL element are dominant at the middle resonance frequency. Because this third resonance has some overlap with the other two, one sees that there also are strong currents flowing on the other two elements.

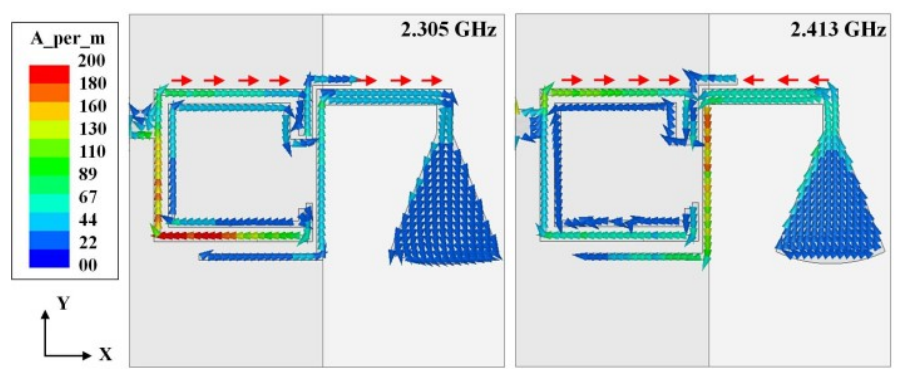

(a)

(b) 


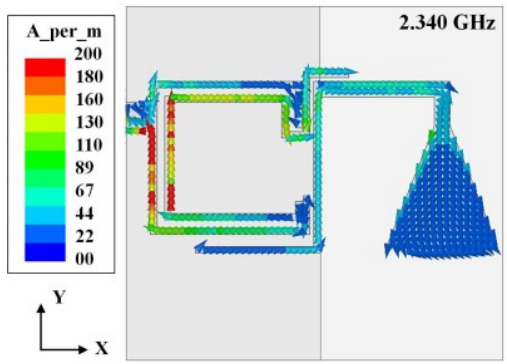

(c)

Fig. 11 The current distribution of the enhanced bandwidth filtenna with slots in its ground strip at the (a) low resonance frequency, $2.305 \mathrm{GHz}$; (b) high resonance frequency, $2.413 \mathrm{GHz}$; and (c) middle resonance frequency, 2.340 $\mathrm{GHz}$.

The simulated and measured directivity patterns at three selected frequencies within the operational band are given in Fig.12. It can be seen that these patterns are relatively the same. Similar to the two-pole filtenna in Fig. 3, the enhanced filtenna with slots in its ground strip radiates a monopole-like pattern in its $E$ - plane and a nearly omni-directional pattern in its $H$-plane. It is observed that the measured $E$-plane patterns have a small amount of tilt from the broadside direction (along $\pm \mathrm{Z}$ axis) and the measured cross polarization levels in the $H$-plane have increased some. These are attributed to the usual unavoidable errors associated with fabrication and measurement, and the presence of the long coaxial cable used in the measurements. On the whole, the experimental results are in good agreement with their simulated values.

\section{CONCLUSIONS}

We have explained the basic design principles to obtain two planar, electrically small, wideband filtennas. The first design evolved from a two-pole filter consisting of a pair of CLL elements. The resulting two-pole filtenna was achieved with a single driven CLL element and an appropriate fan-structured NFRP element that was the primary radiator which replaced the second CLL element of the filter. It was demonstrated that this simple filtenna possesses a flat realized gain response within its operational band and has good band-edge selectivity. To increase the bandwidth while maintaining its steep skirts at the band-edges, a second CLL element was integrated into the system. A majority of it was located inside the region circumscribed by the driven CLL element, while several of its segments were external to that region to achieve the desired resonant length and capacitive coupling levels. It was shown that this additional CLL element alone caused an undesirable ripple in the realized gain values in the middle of the operation band. This ripple was reduced significantly by introducing several slots into the ground strip beneath the CLL elements. Prototypes of both filtennas were fabricated and measured. The simulation and measured results agreed very well.

Based on our current design methodology, the following improvements are anticipated with additional efforts. (1) With the introduction of more CLL elements or some other metamaterial-inspired electrically small resonators that provide the means to control the mutual coupling characteristics, the filtenna impedance bandwidth could be expanded even further. (2) If other compact filters, e.g., from [29], [30], with sharper skirts and wider stopbands are selected as the driven elements of the antenna system, the frequency selectivity of its filtering behavior will be further improved. (3) If a radiative element different from the fan-shaped one could be positioned in the same plane as the driven CLL element and could be designed to act as a near-field parasitic element, it would be possible to construct a compact uniplanar quasi-Yagi configuration and, hence, enable an excellent end-fire radiation performance [31]. (4) Finally, if reconfigurable elements, such as the inclusion of a varactor in the CLL gap, were introduced into the antenna system, its filtering properties could be made tunable, further enhancing its frequency selectivity.

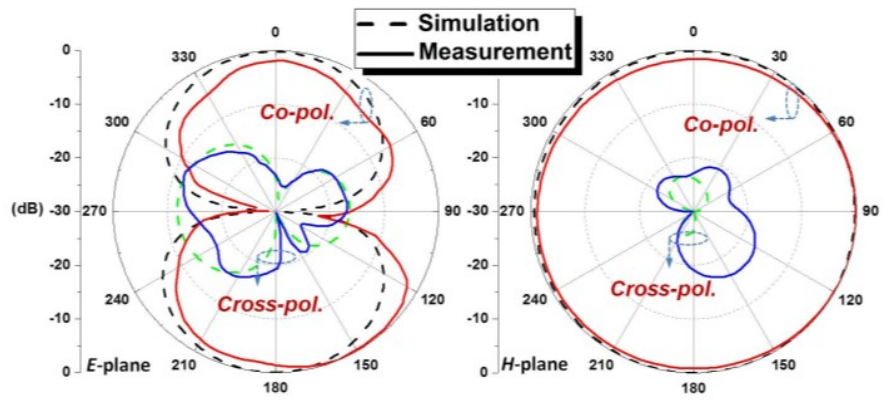

(a)

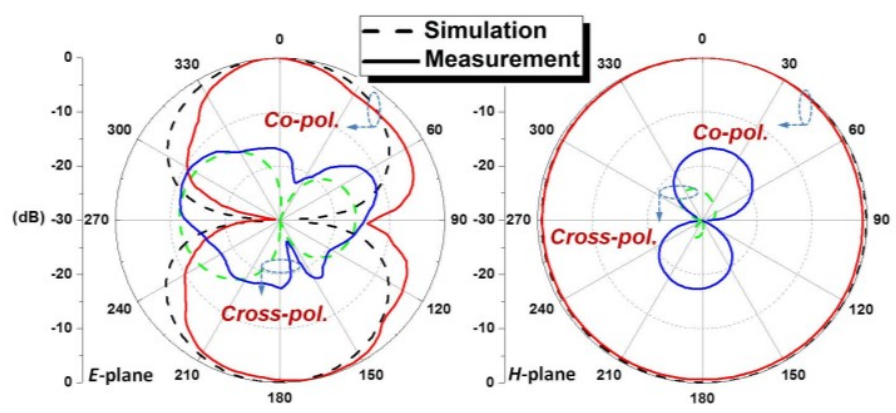

(b)

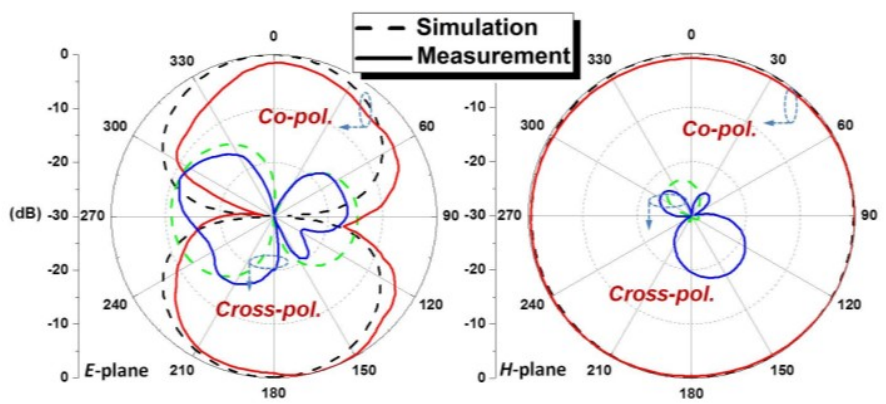

(c)

Fig. 12 The simulated and measured normalized 2D directivity patterns in the $E$ - (ZOX) and $H$ - (ZOY) planes at the three resonant frequencies: (a) 2.305, (b) 2.340 , and (c) $2.413 \mathrm{GHz}$, of the enhanced bandwidth filtenna with slots in its ground strip.

\section{REFERENCES}

[1] G. Q. Luo, W. Hong, H. J. Tang, J. X. Chen, X. X. Yin, Z. Q. Kuai, and K. $\mathrm{Wu}$, "Filtenna consisting of horn antenna and substrate integrated waveguide cavity FSS," IEEE Trans. Antennas Propag., vol. 55, no. 1, pp. 4933-4939, Jan. 2007.

[2] M. Barbuto, F. Trotta, F. Bilotti, and A. Toscano, "Horn antennas with 
integrated notch filters," IEEE Trans. Antennas Propag., vol. 63, no. 2, pp. 781-785, Feb. 2015.

[3] M. Barbuto, F. Trotta, F. Bilotti, and A. Toscano, "Varying the operation bandwidth of metamaterial-inspired filtering modules for horn antennas," Progress In Electromagnetics Research C, vol. 58, pp. 61-68, 2015.

[4] C. Yu, W. Hong, Z. Kuai, and H. Wang, "Ku-band linearly polarized omnidirectional planar filtenna," IEEE Antennas Wirel. Propag. Lett., vol. 11, pp. 310-313, 2012.

[5] O. A. Nova, J. C. Bohórquez, N. M. Peña, G. E. Bridges, L. Shafai, and C. Shafai, "Filter-antenna module using substrate integrated waveguide cavities," IEEE Antennas Wirel. Propag. Lett., vol. 10, pp. 59-62, 2011.

[6] S. W. Wong, T. G. Huang, C. X. Mao, Z. N. Chen, and Q. X. Chu, "Planar filtering ultra-wideband (UWB) antenna with shorting pins," IEEE Trans. Antennas Propag., vol. 61, no. 2, pp. 948-953, Feb. 2013.

[7] M.-C. Tang, T. Shi, and R. W. Ziolkowski, "Planar ultra-wideband antennas with improved realized gain performance," IEEE Trans. Antennas Propag., vol.64, no. 1, pp. 61-69, Jan. 2016.

[8] J. Zuo, X. Chen, G. Han, L. Li, and W. Zhang, "An integrated approach to RF antenna-filter co-design," IEEE Antennas Wirel. Propag. Lett., vol. 8 , pp. 141-144, 2009.

[9] C.-K. Lin and S.-J. Chung, "A compact filtering microstrip antenna with quasi-elliptic broadside antenna gain response," IEEE Antennas Wirel. Propag. Lett., vol. 10, pp. 381-384, 2011.

[10] Z. H. Jiang, and D. H. Werner, "A compact, wideband circularly polarized co-designed filtering antenna and its application for wearable devices with low SAR," IEEE Trans. Antennas Propag., vol.63, no. 9, pp. 3808-3818, Sep. 2015.

[11] X. Y. Zhang, W. Duan, and Y.-M. Pan, "High-gain filtering patch antenna without extra circuit," IEEE Trans. Antennas Propag., vol.63, no. 12, pp. 5883-5888, Dec. 2015.

[12] X. Chen, F. Zhao, L. Yan, and W. Zhang, "A compact filtering antenna with flat gain response within the passband," IEEE Antennas Wirel. Propag. Lett., vol. 12, pp. 857-860, 2013.

[13] J. Shi, X. Wu, Z. N. Chen, X. Qing, L. Lin, J. Chen, and Z.-H. Bao, “A compact differential filtering quasi-Yagi antenna with high frequency selectivity and low cross-polarization levels," IEEE Antennas Wirel. Propag. Lett., vol. 14, pp. 1573-1576, 2015.

[14] L. Chen and Y.-L. Luo, "Compact filtering antenna using CRLH resonator and defected ground structure," Electron. Lett., vol. 51, no. 7, Apr. 2015.

[15] P.-C. Wu, L. Chen and Y.-L. Luo, "Miniaturised wideband filtering antenna by employing CRLH-TL and simplified feeding structure," Electron. Lett., vol. 50, no. 21, Oct. 2014.

[16] C.-T. Chuang and S.-J. Chung, "Synthesis and design of a new printed filtering antenna," IEEE Trans. Antennas Propag., vol. 59, no. 3, pp. 1036-1042, Mar. 2011

[17] W.-J. Wu, Y.-Z. Yin, S.-L. Zuo, Z.-Y. Zhang and J.-J. Xie, "A new compact filter-antenna for modern wireless communication systems," IEEE Antennas Wirel. Propag. Lett., vol. 10, pp. 1131-1134, 2011.

[18] ANSYS/ANSOFT High Frequency Structure Simulation (HFSS), ver. 13.0, ANSYS Corp. [Online]. Available at www.ansoft.com.

[19] C.-M. Tsai, S.-Y. Lee; C.-C. Tsai, "Performance of a planar filter using a $0^{\circ}$ feed structure "IEEE Trans. Microwave Theory Tech., vol. 50, no. 10, pp.2362-2367, Oct. 2002.

[20] J. S. Hong and M. J. Lancaster, Microstrip filters for RF/microwave applications, New York: Wiley, 2001.

[21] S. B. Cohn, "Microwave bandpass filters containing high-Q dielectric resonators," IEEE Trans. Microw. Theory Tech., vol. MTT-16, no. 4, pp. 218-227, Apr. 1968.

[22] Y. Guo and J. Zhou, "Total broadband transmission of microwaves through a subwavelength aperture by localized E-field coupling of split-ring resonators," Opt. Express, vol. 22, no. 22, pp. 27136 - 27143, Nov. 2014

[23] R. W. Ziolkowski, P. Jin, and C.-C. Lin, "Metamaterial-inspired engineering of antennas," Proc. IEEE, vol. 99, no. 10, pp. 1720-1731, Oct. 2011.

[24] StarLab Version D User Guide 1.0, Reference: TD. 224.1.08. SATF. A, SATIMO Corp., 2008, pp. 22-23.

[25] M.-C. Tang, S. Xiao, T. Deng, D. Wang, J. Guan, B.-Z. Wang, and G.-D. Ge, "Compact UWB antenna with multiple band-notches for WiMAX and WLAN," IEEE Trans. Antennas and Propag., vol. 59, no. 4, pp.1372-1376, Apr. 2011.
[26] M.-C. Tang, S. Xiao, D. Wang, G. Ge, and Y.-Y Bai, J. Zhang, and B.-Z. Wang, "Expanding the bandwidth of planar MNG metamaterial with co-directional SRRs," Chin. Phys. B, vol. 20, no. 6, pp. 067805 (1-7), Jun. 2011.

[27] N. Engheta and R.W. Ziolkowski, Metamaterials: Physics and Engineering Explorations, Piscataway, N.J.: IEEE-Wiley Press, 2006.

[28] T. J. Cui, D. R. Smith, and R. Liu, Metamaterials: Theory, Design, and Applications, New York, Springer, 2009

[29] A. Torabi, and K. Forooraghi, "Miniature harmonic-suppressed microstrip bandpass filter using a triple-mode stub-loaded resonator and spur lines," IEEE Microw. Wireless Compon. Lett., vol. 21, no. 5, pp. 255-257, May 2011.

[30] W. Feng, X. Gao, W. Che, and Q. Xue, "Bandpass filter loaded with open stubs using dual-mode ring resonator," IEEE Microw. Wireless Compon. Lett., vol. 25, no. 5, pp. 295-297, May 2015.

[31] M.-C. Tang, T. Shi, and R. W. Ziolkowski, "Flexible efficient quasi-Yagi printed uniplanar antenna," IEEE Trans. Antennas and Propag., vol. 63, no. 12, pp. 5343-5350, Dec. 2015.

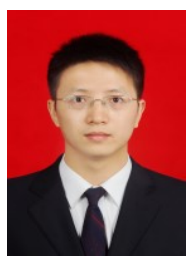

Ming-Chun Tang (S'12-M'13-SM'16) received the B. S. degree in physics from the Neijiang Normal University, Neijiang, China, in 2005 and the $\mathrm{Ph}$. D. degree in radio physics from the University of Electronic Science and Technology of China (UESTC), in 2013. From August 2011 to August 2012, he was also with the Department of Electrical and Computer Engineering, The University of Arizona, Tucson, AZ, USA, as a Visiting Scholar. He is currently an Assistant Professor in the College of Communication Engineering, Chongqing University, China. His research interests include electrically small antennas, RF circuits, metamaterial designs and their applications.

Prof. Tang was a recipient of the Best Student Paper Award in the 2010 International Symposium on Signals, Systems and Electronics (ISSSE2010) held in Nanjing, China. He is serving as a reviewer for several IEEE/IET journals.

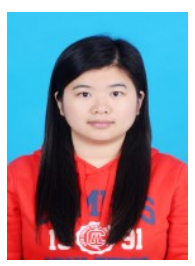

Ying Chen received the B.S. degree from the University of Electronic Science and Technology of China (UESTC), Chengdu, China, in 2014. She is currently pursuing the M.S. degree in electronics and communication engineering in the College of Communication Engineering, Chongqing University, China.

Her current research interests include electrically small antenna and filtenna designs and their applications.

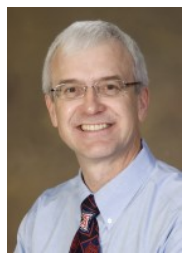

Richard W. Ziolkowski (M'87-SM'91-F'94) received the the B.Sc. degree (magna cum laude) with Honors from Brown University, Providence, RI, USA, in 1974, and the M.S. and Ph.D. degrees from the University of Illinois at Urbana-Champaign, Urbana, IL, USA, in 1975 and 1980, respectively, all in physics, and the Doctor Technish Honoris Causa degree from the Technical University of Denmark, Kongens Lyngby, Denmark, in 2012.

He was the Computational Electronics and Electromagnetics Thrust Area Leader with the Lawrence Livermore National Laboratory, Engineering Research Division, Livermore, CA, USA, before joining The University of Arizona, Tucson, AZ, USA, in 1990. He is currently the Litton Industries John M. Leonis Distinguished Professor with the Department of Electrical and Computer Engineering, The University of Arizona, where he is also a Professor with the College of Optical Sciences. He is also a Distinguished Professor with the Global Big Data Technologies Centre at the University of Technology Sydney, Ultimo NSW, Australia.

Prof. Ziolkowski is a fellow of the Optical Society of America (OSA). He is actively involved with the URSI, OSA, and SPIE professional societies. He served as the President of the IEEE Antennas and Propagation Society in 2005. He was the Australian Defence Science and Technology Organisation Fulbright Distinguished Chair in Advanced Science and Technology from 2014 to 2015. 